\title{
Toward an accurate description of solid-state properties of superheavy elements
}

\section{A case study for the element $\mathrm{Og}(Z=118)$}

\author{
Peter Schwerdtfeger ${ }^{\mathrm{a}}$ \\ Centre for Theoretical Chemistry and Physics, The New Zealand Institute for Advanced Study, Massey \\ University (Albany Campus), Auckland, New Zealand
}

\begin{abstract}
In the last two decades cold and hot fusion experiments lead to the production of new elements for the Periodic Table up to nuclear charge 118. Recent developments in relativistic quantum theory have made it possible to obtain accurate electronic properties for the trans-actinide elements with the aim to predict their potential chemical and physical behaviour. Here we report on first results of solid-state calculations for Og (element 118) to support future atom-at-a-time gas-phase adsorption experiments on surfaces such as gold or quartz.
\end{abstract}

\section{Introduction}

Mendeleev arranged the known elements (60 in 1869) into a Periodic Table according to their atomic weights and recurring chemical properties. A proper foundation for this ordering scheme came through the electronic shell model of Bohr, and later through Schrödinger's quantum theory providing proper assignments for the ground state electron configurations of the elements. The Periodic Table now contains 118 known and named elements finishing with the $7 p$ electronic shell closure at the recently discovered rare gas element 118 (Oganesson, $\mathrm{Og}$ ) [1]. Predictions on the placements of even heavier elements have been made recently by Pyykkö [2,3] for nuclear charges up to $Z=172^{1}$ (see also earlier work by Fricke [4]), although attempts to synthesize elements beyond nuclear charge 118 have been unsuccessful so far.

The newest elements in the Periodic Table are rather short-lived undergoing predominantly $\alpha$-decay within the milli-second to second range [5, 6]. Lifetimes in the second range or above open up the possibility for atom-at-a-time experiments to study the chemical and physical behaviour of such exotic elements [7-9]. For this one requires more neutron-rich superheavy element isotopes near the neutron shell closure at $N=184$. Future experiments with neutron-rich projectiles or targets might yield longer lived isotopes to study the chemistry of the heaviest elements such as Og. Physisorption studies on gold surfaces already gave estimates for adsorption enthalpies for the superheavy elements $112(\mathrm{Cn})$ and $114(\mathrm{Fl})$. According to an early study of Eichler et al., these adsorption enthalpies correlate well with the cohesive energies of the bulk elemental substances [10]. It is therefore of current interest to estimate the cohesive energies for such superheavy elements.

\footnotetext{
${ }^{a}$ e-mail: p.a.schwerdtfeger@massey .ac.nz

${ }^{1}$ At such high nuclear charges the mean field picture of the Dirac equation starts to breaks down [12].
} 
It is currently a challenge for computational chemistry to obtain accurate lattice parameters and cohesive energies for bulk systems [11], especially for the heavier elements where relativistic and electron correlation effects compete with each other [12-15]. For the rare gas elements, the atomic interaction is of dispersive nature. The total energy of the crystal can therefore be decomposed into the individual many-body interaction terms [16, 17]. At long range such a many-body decomposition converges fast and one already obtains correct trends in cohesive energies along the rare gas series of elements from a simple sum over diatomic contributions [19]. Using such an incremental scheme, Stoll and co-workers were able to obtain accurate cohesive energies for the rare gas fcc crystals of neon, argon, krypton and xenon $[20,21]$. Here we present preliminary results using a simple Lennard-Jones (LJ) potential for the two-body term and an Axilrod-Teller (AT) three-body ansatz [18] for the three-body term to obtain first estimates for cohesive energies of the rare gas fcc crystals Rn and $\mathrm{Og}$.

\section{Theory}

The cohesive energy of a rare gas crystals can be written as a series of $n$-body interaction terms (called the incremental method) [16, 17],

$$
E_{\mathrm{coh}}\left(r_{s}\right) \cong \Delta E_{\mathrm{coh}}^{(2)}\left(r_{s}\right)+\Delta E_{\mathrm{HE}-\mathrm{ZPE}}^{(2)}\left(r_{s}\right)+\Delta E_{\mathrm{AE}-\mathrm{ZPE}}^{(2)}\left(r_{s}\right)+\Delta E_{\mathrm{coh}}^{(3)}\left(r_{s}\right)+\ldots
$$

Here $r_{s}$ is the nearest neighbour distance in the solid, and $E^{(n)}$ is the $n$-body contribution to the cohesive energy [26],

$$
\Delta E_{\mathrm{coh}}^{(2)}\left(r_{s}\right)=\frac{1}{2} \epsilon L_{12}\left(\frac{r_{e}}{r_{s}}\right)^{12}-\epsilon L_{6}\left(\frac{r_{e}}{r_{s}}\right)^{6}
$$

and

$$
\Delta E_{\mathrm{coh}}^{(3)}\left(r_{s}\right)=\frac{1}{3} C_{\mathrm{AT}} \sum_{j<k}\left(1+3 \cos \theta_{0} \cos \theta_{j} \cos \theta_{k}\right)\left(r_{0 j} r_{0 k} r_{j k}\right)^{-3}
$$

where $r_{i j}$ is the distance between atoms $i$ and $j$ in the crystal, $\theta_{i}$ is the angle between the vectors $\vec{r}_{i j}$ and $\vec{r}_{i k}$, and $C_{\mathrm{AT}}$ is the Axilrod-Teller coefficient approximated by known or calculated atomic static dipole polarizabilities $\alpha$ and ionization potentials $E_{\mathrm{IP}}$ [27, 28], i.e. we have,

$$
C_{\mathrm{AT}}=f_{\mathrm{AT}} \alpha^{3} E_{\mathrm{IP}}
$$

The prefactors $f_{\mathrm{AT}}$ for $\mathrm{Rn}$ and $\mathrm{Og}$ are obtained from an extrapolation of known values for the lighter rare gases. We sum from a chosen atom placed at the origin to all other atoms in the lattice at distance $r_{0 j}$ with the nearest neighbour distance defined as $r_{s}=\min _{j}\left\{r_{0 j}\right\} . L_{6}$ and $L_{12}$ are the Lennard-Jones-Ingham coefficients for the fcc crystals given in Ref. [30]. For the zero-point vibrational energies (ZPE) to the two-body cohesive energy we used recently derived analytical expressions for the harmonic (HE) and anharmonic (AE) Einstein contributions (the latter derived from first-order perturbation theory) [29]. The optimization of the lattice geometry has been performed with our program system SAMBA [30].

\section{Results}

The results are shown in Tables 1 and 2, and summarized for the cohesive energies in Fig. 1. For the lighter rare gas solids vibrational effects are important whilst for the heavier systems the three-body force cannot be neglected anymore. For bulk Ne to Xe our results are in reasonably good agreement with the available experimental data. Deviations are mainly due 
Table 1. Optimized bond distances for the rare gas fcc lattices from Ne to $\mathrm{Og}(Z=118)$ using a Lennard-Jones (6-12) potential and a three-body Axilrod-Teller term. Equilibrium distances $r_{e}$ of the diatomic taken from references [22-25], and nearest neighbour distance of the solid $r_{s}^{\mathrm{min}}$ at various levels of theory (see text). The total ZPE (TZPE) includes both harmonic (HE) and anharmonic (AE) terms. All values in $[\AA]$, atomic masses $M$ in [u]. Experimental solid-state distances are from Refs. [31-35].

\begin{tabular}{r|rrrrrrr} 
Element & \multicolumn{1}{c}{$M$} & \multicolumn{1}{c}{$r_{e}$} & $r_{s}^{\mathrm{LJ}}$ & $r_{s}^{\mathrm{LJ}+\mathrm{HE}-\mathrm{ZPE}}$ & $r_{s}^{\mathrm{LJ}+\mathrm{TZPE}}$ & $r_{s}^{\mathrm{LJ}+\mathrm{TZPE}+\mathrm{AT}}$ & $r_{s}^{\text {exp }}$ \\
\hline${ }^{20} \mathrm{Ne}$ & 19.992 & 3.089 & 3.001 & 3.125 & 3.120 & 3.134 & 3.157 \\
${ }^{40} \mathrm{Ar}$ & 39.962 & 3.762 & 3.654 & 3.697 & 3.697 & 3.731 & 3.756 \\
${ }^{84} \mathrm{Kr}$ & 83.912 & 4.016 & 3.900 & 3.925 & 3.925 & 3.968 & 4.009 \\
${ }^{132} \mathrm{Xe}$ & 131.904 & 4.363 & 4.237 & 4.254 & 4.254 & 4.310 & 4.336 \\
${ }^{222} \mathrm{Rn}$ & 222.018 & 4.427 & 4.300 & 4.310 & 4.310 & 4.367 & - \\
${ }^{294} \mathrm{Og}$ & 294.000 & 4.329 & 4.204 & 4.211 & 4.211 & 4.282 & - \\
\hline
\end{tabular}

Table 2. Cohesive energies at optimized bond distances (see Table 1) for the rare gas fcc lattices from Ne to $\mathrm{Og}(Z=118)$ using a Lennard-Jones (6-12) potential and a three-body Axilrod-Teller term. Lennard-Jones binding energies $-\epsilon$ (taken from references [22-25]) and cohesive energies $E^{\mathrm{coh}}$ at various levels of theory (see text). The total ZPE (TZPE) includes both harmonic (HE) and anharmonic terms (AE). All values are in [meV]. Experimental cohesive energies are from references [35-37].

\begin{tabular}{r|crrrrr} 
Element & $-\epsilon$ & $E_{\text {coh }}^{\mathrm{LJ}}$ & $E_{\text {coh }}^{\mathrm{LJ}+\mathrm{HE}-\mathrm{ZPE}}$ & $E_{\text {coh }}^{\mathrm{LJ}+\mathrm{TZPE}}$ & $E_{\text {coh }}^{\mathrm{LJ}+\mathrm{TZPE}+\mathrm{AT}}$ & $E_{\text {coh }}^{\text {exp }}$ \\
\hline${ }^{20} \mathrm{Ne}$ & -3.63 & -31.3 & -23.6 & -22.9 & -22.2 & -20.5 \\
${ }^{40} \mathrm{Ar}$ & -12.3 & -106.2 & -96.9 & -96.7 & -90.1 & -80.0 \\
${ }^{84} \mathrm{Kr}$ & -17.3 & -149.0 & -141.7 & -141.6 & -130.3 & -116.0 \\
${ }^{132} \mathrm{Xe}$ & -24.3 & -209.4 & -203.0 & -203.0 & -183.3 & -164.7 \\
${ }^{222} \mathrm{Rn}$ & -34.9 & -300.4 & -294.5 & -294.5 & -265.8 & $(-202)$ \\
${ }^{294} \mathrm{Og}$ & -77.4 & -666.4 & -658.6 & -658.5 & -577.3 & - \\
\hline
\end{tabular}

to the fact that we chose a simple Lennard-Jones two-body potential, the neglect of phonon dispersion and higher order terms in our many-body decomposition. This suggests that our predictions for the properties of the heaviest rare gas solids, $\mathrm{Rn}$ and $\mathrm{Og}$, should be reasonably accurate as well.

The nearest neighbour distances $r_{s}$ and cohesive energies $E_{\mathrm{coh}}\left(r_{s}\right)$ are dependent on our chosen Lennard-Jones parameter in Eq. (6), that is the bond distance $r_{e}$ and the binding energy $\epsilon$. This can be seen from simple relationships known for Lennard-Jones interactions [30],

$$
r_{s}^{\mathrm{LJ}}=\left(\frac{L_{12}}{L_{6}}\right)^{1 / 6} r_{e}
$$

and

$$
E_{\mathrm{coh}}\left(r_{s}^{\mathrm{LJ}}\right)=\frac{1}{2}\left(\frac{L_{6}^{2}}{L_{12}}\right) \epsilon
$$

We see that the short bond distance for the Og dimer (Table 1) [24] is causing an anomalous behaviour in the trend down the rare gas dimers. This translates directly into an anomaly in the nearest neighbour distances of the rare gas solids down that group. The bond distances $r_{e}$ and binding energies $\epsilon$ for $\mathrm{Rn}_{2}$ and $\mathrm{Og}_{2}$ were taken from relativistic coupled cluster calculations of Saue and co-workers, which include corrections for basis set superposition errors and are extrapolated to the basis set limit [24]. Their perturbative triples contributions to the dissociation energy of the rare gas dimers in the coupled cluster procedure are however rather large indicating that one has to include quadruple corrections as well within the coupled cluster procedure, which becomes computationally demanding. However, their CCSD(T) 


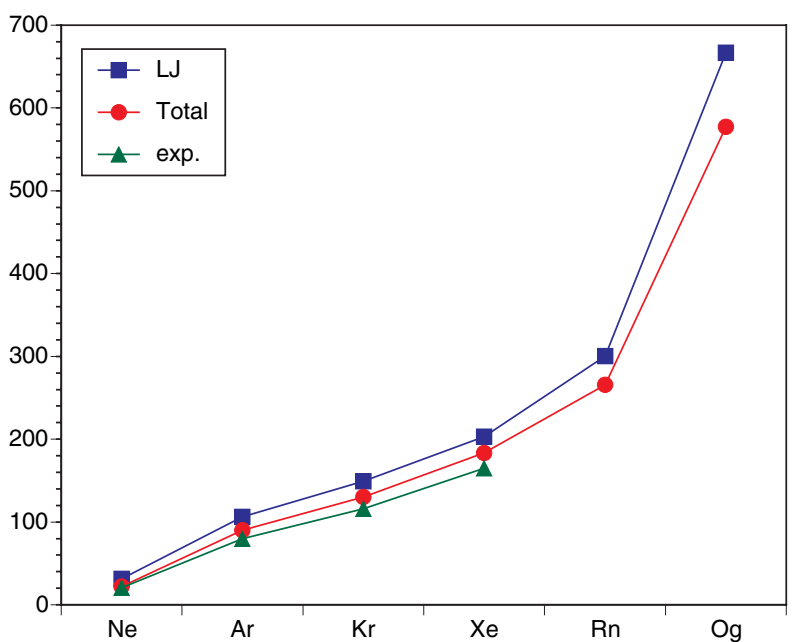

Figure 1. Cohesive energies $-E_{\text {coh }}$ (total) in $[\mathrm{meV}]$ for the rare gas crystals in comparison to the available experimental values. The pure two-body Lennard-Jones (LJ) contribution is shown as well for comparison.

results for the bond distance and the dissociation energy of $\mathrm{Xe}_{2}$ are in good agreement with experiment [35]. We note that our cohesive energies for the bulk systems are overestimated by $8.0 \%$ for $\mathrm{Ne}$, to $12.6 \%$ for $\mathrm{Ar}, 11.2 \%$ for $\mathrm{Kr}$, and $11.1 \%$ for $\mathrm{Xe}$ due to the various approximations made in this preliminary study. Taking this into account for the heavier rare gas solids our best estimates for the cohesive energies are 230 and $500 \mathrm{meV}$ for $\mathrm{Rn}$ and $\mathrm{Og}$, respectively. The cohesive energy for $\mathrm{Og}$ is unusually large for a rare gas element and comparable to the estimated cohesive energies of $\mathrm{Cn}\left(390\left({ }_{-100}^{+120}\right) \mathrm{meV}\right)$ [38-40] and $\mathrm{Fl}$ (500 meV from theory [41] and $220\left({ }_{-20}^{+230}\right) \mathrm{meV}$ from experiment [42, 43]). Eichler and Eichler predicted thermochemical data for the superheavy elements empirically [44]. Their estimated cohesive energy is half our predicted value $(\sim 250 \mathrm{meV})$, but interestingly is larger than their empirical data for $\mathrm{Cn}$ and $\mathrm{Fl}$.

\section{Conclusions}

We presented preliminary calculations for the rare gas solids up to the heaviest element $\mathrm{Og}$. The cohesive energy of $\sim 500 \mathrm{meV}$ for $\mathrm{Og}$ was calculated to be unusually large when compared to the other rare gas solids, which is most likely caused by the strong spin-orbit splitting in the $7 p$ shell making the $7 p_{3 / 2}$ orbital rather diffuse. This leads to an increased dipole polarizability for $\mathrm{Og}$ (59.0 a.u.) [47] compared to $\mathrm{Rn}$ (33.2 a.u.) [48], for example, thus increasing dispersive type of interactions and causing an anomaly in the trend of nearest neighbour distances for the bulk rare gas systems. Hence in this respect $\mathrm{Og}$ is not so inert anymore (NB: it is the first rare gas element with a positive electron affinity [45, 46]). We expect that bulk Og melts at much higher temperatures compared to the lighter rare gas elements. More accurate quantum chemical calculations for the potential energy curves and the Axilrod-Teller term of the heaviest rare gas dimers are however required to produce better cohesive energies. Relativistic effects (including and spin-orbit coupling) as a cause for this steep increase from $\mathrm{Rn}$ to $\mathrm{Og}$ in the cohesive energy need to be analyzed as well. Work in this direction is currently in progress. 
This work was supported by a Marsden grant administered by the Royal Society of New Zealand. We thank Dr. Robert Eichler and Prof. Heinz Gäggeler for valuable discussions.

\section{References}

[1] Here we adopt the suggested IUPAC name for element 118, Oganesson. See http: //iupac.org/.

[2] P. Pyykkö, Phys. Chem. Chem. Phys. 13, 161 (2011).

[3] P. Pyykkö, Chem. Rev. 112, 371 (2011).

[4] B. Fricke, in Structure and Bonding (Springer, Berlin, Vol. 21, 1975), p. 89.

[5] Yu.Ts. Oganessian et al., Phys. Rev. C 74, 044602 (2006).

[6] Yu.Ts. Oganessian et al., Phys. Rev. Lett. 104, 142502 (2010).

[7] M. Schädel (ed.), The Chemistry of Superheavy Elements (Kluwer Academic, Dordrecht, 2003).

[8] V. Pershina, Chem. Rev. 96, 1977 (1997).

[9] A. Türler and V. Pershina, Chem. Rev. 113, 237 (2013).

[10] R. Eichler, Radiochim. Acta 93, 245 (2005).

[11] P. Fulde, Electron Correlations in Molecules and Solids (Springer Series in Solid-State Sciences, Springer, Heidelberg, 1995).

[12] P. Schwerdtfeger, L.F. Pašteka, A. Punnett, P. Bowman, Nucl. Phys. A944, 551 (2015).

[13] P. Pyykkö, Chem. Rev. 88, 563 (1988).

[14] E. Eliav, U. Kaldor, P.Schwerdtfeger, B.A. Hess, Y.Ishikawa, Phys. Rev. Lett. 73, 3203 (1994).

[15] P. Schwerdtfeger and M. Seth, in The Encyclopedia of Computational Chemistry, P. von R. Schleyer, N.L. Allinger, T. Clark, J. Gasteiger, P. Kollman, H.F. Schaefer III (eds.), Wiley, New York, 1998, p. 2480.

[16] H. Stoll, Phys. Rev. B 46, 6700 (1992).

[17] B. Paulus, Phys. Rep. 428, 1 (2006).

[18] B.M. Axilrod and E. Teller, J. Chem. Phys. 11, 299 (1943).

[19] A. Hermann, R.P. Krawczyk, M. Lein, P. Schwerdtfeger, I.P. Hamilton, J.J.P. Stewart, Phys. Rev. A 76, 013202 (2007).

[20] K. Rosćiszewski, B. Paulus, P. Fulde, H. Stoll, Phys. Rev. B 60, 7905 (1999).

[21] K. Rosćiszewski, B. Paulus, P. Fulde, H. Stoll, Phys. Rev. B 62, 5482 (2000).

[22] E. Bich, R. Hellmann, E. Vogel, Mol. Phys. 106, 1107 (2008).

[23] B. Jäger, R. Hellmann, E. Bich, E. Vogel, Mol. Phys. 107, 2181 (2009).

[24] A. Shee, S. Knecht, T. Saue, Phys. Chem. Chem. Phys. 17, 10978 (2015).

[25] P. Slavíček, R. Kalus, P. Paška, I. Odvárková, P. Hobza, A. Malijevský, J. Chem. Phys. 119, 2102 (2003).

[26] J.E. Lennard-Jones and A.E. Ingham, Proc. Roy. Soc. A 107, 636 (1925).

[27] C.E. Moore, Atomic Energy Levels; Natl. Bur. Stand. (U.S.) Circ. No. 467; U.S. GPO: Washington D.C., 1958.

[28] P. Schwerdtfeger, Table of experimental and calculated static dipole polarizabilities for the electronic ground states of the neutral elements (CTCP Auckland, 2016); available at http://ctcp.massey.ac.nz/index.php?group=\&page=dipole\& menu=dipole.

[29] P. Schwerdtfeger, A. Burrows, E. Pahl, unpublished results.

[30] P. Schwerdtfeger, N. Gaston, R.P. Krawczyk, R. Tonner, G.E. Moyano, Phys. Rev. B 73, 064112 (2006).

[31] C.S. Barrett and L. Meyer, J. Chem. Phys. 41, 1078 (1964). 
[32] O.G. Peterson, D.N. Batchelder, R.O. Simmons, Phys. Rev. 150, 7031966.

[33] D.N. Batchelder, D.L. Losee, R.O. Simmons, Phys. Rev. 162, 767 (1967).

[34] D.L. Losee and R.O. Simons, Phys. Rev. 172, 944 (1968).

[35] J. Ogilvie and F.Y. Wang, J. Mol. Struct. 273, 277 (1992).

[36] G.J. McConville, J. Chem. Phys. 60, 4093 (1974).

[37] L.A. Schwalbe, R.K. Crawford, H.H. Chen, R.A. Aziz, J. Chem. Phys. 66, 4493 (1977).

[38] R. Eichler et al., Nature 447, 72 (2007).

[39] N. Gaston, I. Opahle, H.W. Gäggeler, P. Schwerdtfeger, Angew. Chem. Int. Ed. 46, 1663 (2007).

[40] R. Eichler et al., Angew. Chem. Int. Ed. 47, 3262 (2008).

[41] A. Hermann, H. Gäggeler, P. Schwerdtfeger, Phys. Rev. B 82, 155116 (2010).

[42] R. Eichler et al., Radiochim. Acta 98, 133 (2010).

[43] A. Yakushev et al., Inorg. Chem. 53, 1624 (2014).

[44] R. Eichler and B. Eichler, in The Chemistry of Superheavy Elements, M. Schädel and D. Shaughnessy (eds.), (Springer, Heidelberg, 2014), p. 375.

[45] E. Eliav, U. Kaldor, Y. Ishikawa, P. Pyykkö, Phys. Rev. Lett. 77, 5350 (1996).

[46] I. Goidenko, L. Labzowsky, E. Eliav, U. Kaldor, P. Pyykkö, Phys. Rev. A 67, 020102(R) (2003).

[47] V. Pershina, A. Borschevsky, E. Eliav, U. Kaldor, J. Chem. Phys. 129, 144106 (2008).

[48] T. Nakajima and K. Hirao, Chem. Lett. (Jpn.) 8, 766 (2001). 